\title{
APPLYING FUZZY AHP TO EVALUATE THE CARBON FOOT PRINT ON THE WORKPLACE IN EDUCATIONAL INSTITUTIONS
}

\author{
Shyamsundar $\mathbf{R}^{1}$, Selvakumar $\mathbf{A}^{2}$, Visagavel $\mathbf{K}^{3}$ \\ ${ }^{I}$ PG Scholar, Department of Mechanical Engineering, Knowledge Institute of Technology, Tamilnadu, India \\ ${ }^{2}$ Assistant Professor, Department of Mechanical Engineering, Knowledge Institute of Technology, Tamilnadu, India \\ ${ }^{3}$ Professor, Department of Mechanical Engineering, Knowledge Institute of Technology, Tamilnadu, India
}

\begin{abstract}
Laboratories in educational institutions needs methods to determine the heat emitted from those in a simple manner. The objective of this study was to construct a practical approach based on "fuzzy Analytic Hierarchy Process"(FAHP) for selecting the least heat emission laboratory. In this paper 5 alternatives and 5 criteria are considered which are from the experts "Knowledge and Judgments". Fuzzy Analytic Hierarchy Process applied for calculating relative weights of each criterion. It is the effective assessment tool for selecting the least heat emission laboratory. The analyzed results had identified the relative weight of the criteria taken in laboratory and heat emissions in different laboratories were compared. Thus the study results will provide a practical reference for the persons engaged in the work in laboratory.
\end{abstract}

Keywords: Heat emission from laboratories, fuzzy analytic hierarchy process, multi criteria decision making. ****.

\section{INTRODUCTION}

The institutional laboratories have containing much electronic equipment and are increasing heat emission to the environment. The increasing number of users using the equipment results in the higher heat emitted from the equipment's. As a consequence of an increasing equipment's used in laboratories, the persons engaged in the laboratories have been faced an higher thermal condition environment. They may not aware of amount of thermal conditions inside the laboratory. For taking as a reference this study will provide a practical reference for them. Hence the selection of least heat emitted laboratory is an important factor that determines the thermal condition in that.

Evaluating and selecting the least heat emission laboratory can be regarded as multi criteria decision making(MCDM)process in which the users and decision makers chooses under the several selection criteria, [2] the best thermal condition of laboratory among the alternatives.The MCDM is an Analytic hierarchy process (AHP) is introduced by saaty(1980). A lot of researchers have applied AHP to solve many MCDM process in various areas such as project selection, finance management, performance evaluation, economic planning.

This research involves the process of identifying important criteria that should be considered in terms of selecting the alternatives of laboratories in educational institutions. Then next process is to apply Fuzzy AHP for calculating weights and importance of each criterion and rank the alternatives.

\section{LITERATURE REVIEW OF FUZZY AHP}

The calculation of AHP is based on the methods and concepts developed by Buckley (1985) and the trapezoidal fuzzy numbers (TrFN) of Buckley (1985) are substituted by Triangular fuzzy numbers.

AHP involves three principles to solve the problems. (SarfarazHashemkhani et al., 2012).

1. Structure of the hierarchy.

2. The matrix of pair wise comparison ratios.

3. The method for calculating weights.

AHP can breakdown any complex problems into several parts in terms of hierarchical level where the criteria are selected for each level of hierarchy to each parts of problem.

- Develop the hierarchical structure for the problem of investigation and derive a fuzzy pairwise comparison matrix.

- Calculate the relative weights and fuzzy positive reciprocal matrix.

- Defuzzification: conversion of fuzziness into exact values is defined as defuzzification. (Normalization and the hierarchy coordination.

Many methods and applications of Fuzzy AHP are expressed by various researchers[1]. The FAHP method is used to determine the relative weights of criteria for decision makers by individual opinion. This method is chosen to calculate the weights of selected methods in order to reduce the fuzziness and ambiguity of information and ranking. It displays that the Fuzzy AHP method for selecting the thermal condition of laboratory can be useful and effective assessment tool. 


\section{METHODOLOGY}

Over the years the complexity of heat emission in a laboratory has rapidly increases, thus identifying the importance of developing and implementing practical and effective quantitative analysis techniques for evaluation of least heat emission laboratory in educational institutions.(MCDM) technique is an advanced research operation, gives decision makers and analysts a broad range.

To investigate a number of alternatives in lights of conflicting priorities multi criteria analysis (MCA) gives a structure for breaking a problem into its constituent parts. Multi criteria are selected based on the knowledge of experts and data collected from the institutional laboratories. Therefore this study aims to use FAHP to evaluate the heat emission in laboratory which serves as a basis of reference and rank the least heat emitted laboratory among the alternatives.

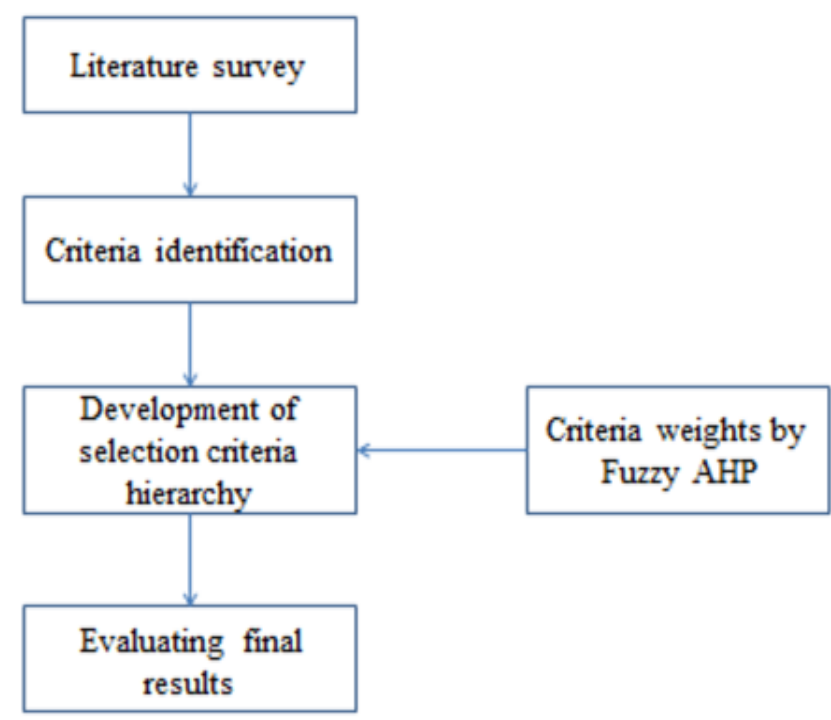

Fig1: Framework of research

\subsection{Fuzzy Analytic Hierarchy Process}

In (1983) FAHP method was developed by Laarhoven and Pedrycz based on the AHP method proposed by Saaty (1980) [4]. FAHP has been widely engaged in number of different issues which can define and analysesthe problems efficiently. Therefore the FAHP method is used in this study to find out the weights of each criterion selected for evaluation of least heat emitted thermal conditional laboratory.

\subsection{Criteria Selection}

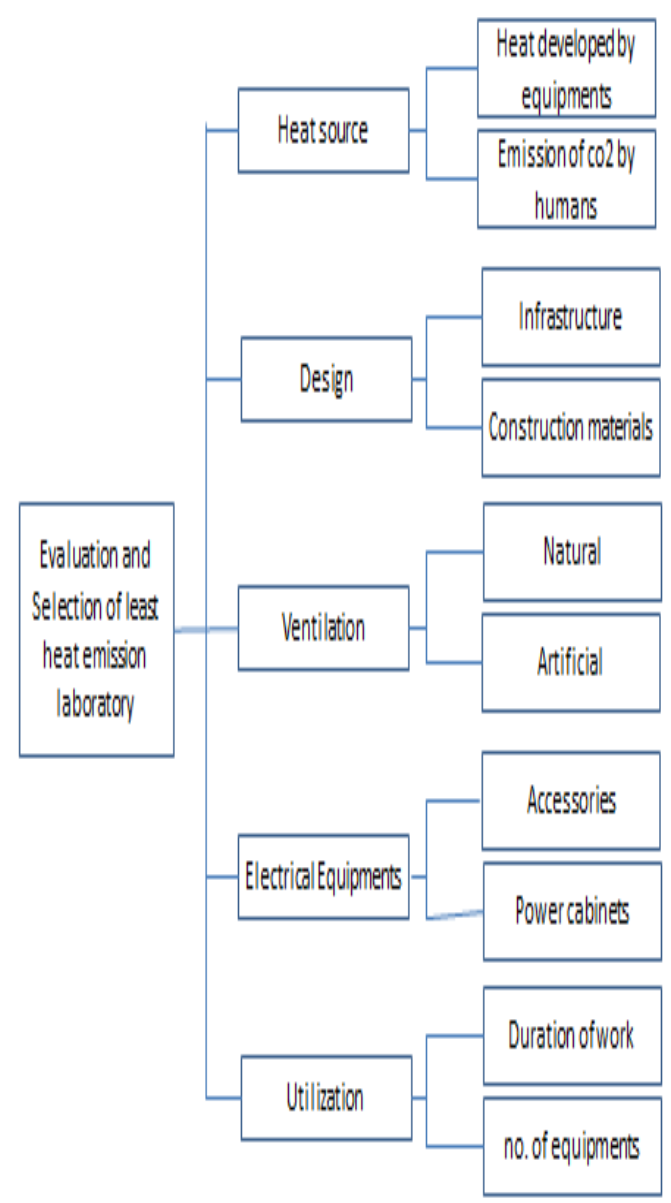

Fig2: Research hierarchical structure

\subsection{Application}

The objective of this paper is to choose ideal institutional laboratory based on fuzzy AHP method. In this research , five alternatives and five criteria have been plotted. Alternatives (A1), Cad lab(A2), Cam lab(A3), Electronics lab(A4), and Electrical lab(A5). The motive behind for selecting these alternatives is the highest heat emitting workplace in educational institutions. This study criterion includes Heat source(C1), Design(C2), Ventilation(C3), Electrical Equipment's(C4), and Utilization(C5). These criteria through interviews with workplace authorities and experts has been selected and taken out. Then the pairwise comparisons surveyresulting to criteria and alternatives were provided. Fuzzy AHP method was used to weight the criteria and alternatives. After this method, the best Laboratory has determined. According to the criteria and alternatives, the research hierarchical structure has shown in figure 3 .

As cause of variance between experts' judgments and for minimizing vagueness and uncertainty in decision making process, Fuzzy AHP method is suggest for group decision making process. According to the table 1 each decision maker independently was using pairwise comparison based on Saaty's 1-9 scale [1]. 


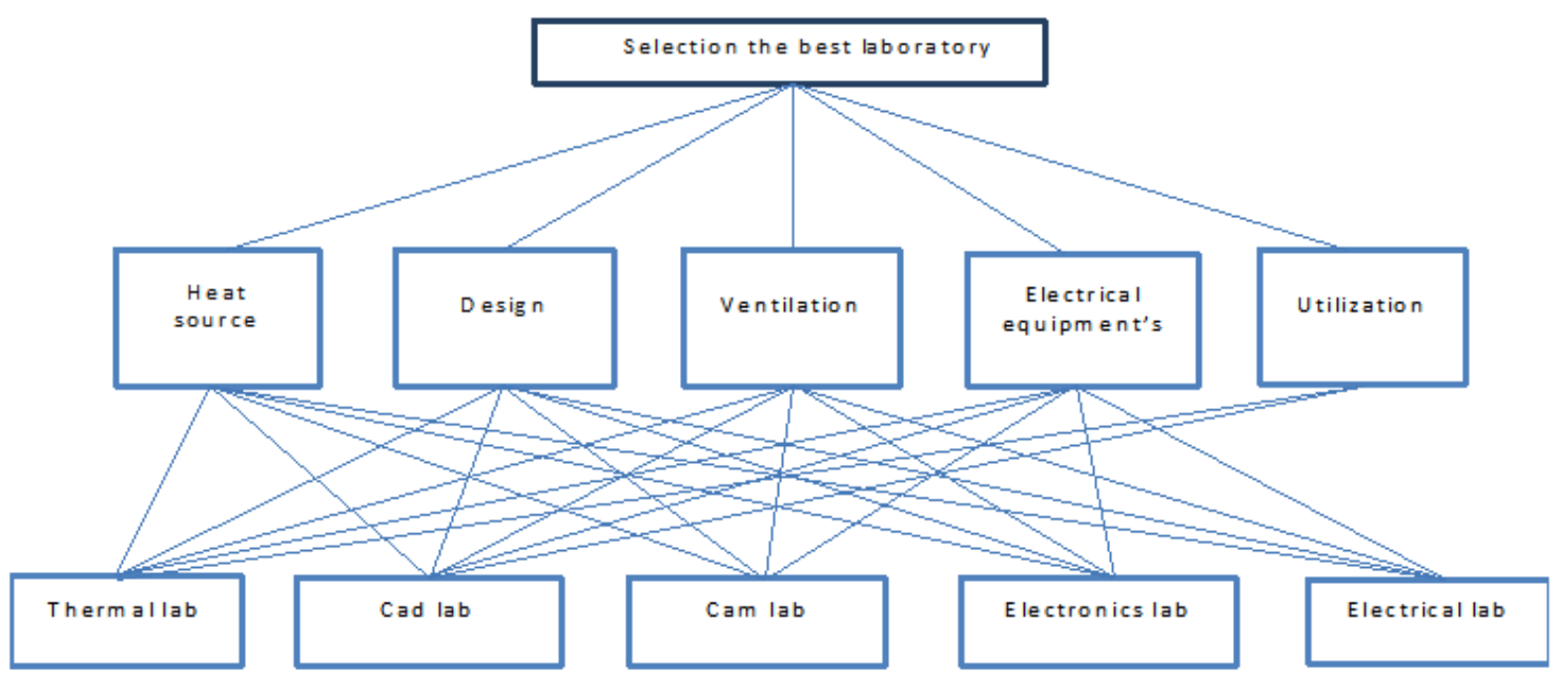

Fig 3: The hierarchy structure of selection green laboratory in educational institution

Table 1: Pair-wise comparison scale (Saaty, 1980)

\begin{tabular}{|l|l|l|l|l|l|l|}
\hline Fuzzy number & 1 & 3 & 5 & 7 & 9 & $2,4,6,8$ \\
\hline Definition & $\begin{array}{l}\text { Equally } \\
\text { important }\end{array}$ & $\begin{array}{l}\text { Moderately } \\
\text { more important }\end{array}$ & $\begin{array}{l}\text { Strongly more } \\
\text { important }\end{array}$ & $\begin{array}{l}\text { Very strongly } \\
\text { more } \\
\text { Important }\end{array}$ & $\begin{array}{l}\text { Extremely } \\
\text { more important }\end{array}$ & $\begin{array}{l}\text { Intermediate } \\
\text { values } \\
\text { Between the } \\
\text { two adjacent } \\
\text { Judgments. }\end{array}$ \\
\hline
\end{tabular}

\subsection{Calculation}

Table 2: Fuzzy criteria weight number for alternatives

\begin{tabular}{|l|l|l|l|l|l|l|l|l|}
\hline & $\begin{array}{l}\text { Heat } \\
\text { source }\end{array}$ & Design & Ventilation & $\begin{array}{l}\text { Electrical } \\
\text { equipment }\end{array}$ & Utilization & Product & $\begin{array}{l}\text { 5th root of } \\
\text { product }\end{array}$ & $\begin{array}{l}\text { Normalized } \\
\text { weight }\end{array}$ \\
\hline $\begin{array}{l}\text { Heat } \\
\text { source }\end{array}$ & 1 & 7 & 5 & 7 & 9 & 2205 & 4.66318 & 0.59769 \\
\hline Design & $1 / 7$ & 1 & 3 & 5 & 1 & 2.14286 & 1.16466 & 0.14928 \\
\hline Ventilation & $1 / 5$ & $1 / 3$ & 1 & 7 & 5 & 2.33333 & 1.18466 & 0.15184 \\
\hline $\begin{array}{l}\text { Electrical } \\
\text { equip }\end{array}$ & $1 / 7$ & $1 / 5$ & $1 / 7$ & 1 & 3 & 0.01224 & 0.41456 & 0.05314 \\
\hline Utilization & $1 / 9$ & 1 & $1 / 5$ & $1 / 3$ & 1 & 0.00741 & 0.37492 & 0.04805 \\
\hline $\begin{array}{l}\text { Sum of the } \\
\text { coloumn }\end{array}$ & 1.5968 & 9.5333 & 9.3429 & 20.3333 & 19.0000 & & 7.8020 & \\
\hline $\begin{array}{l}\text { Sum } \\
\text { normalized } \\
\text { weight }\end{array}$ & 0.9544 & 1.4231 & 1.4186 & 1.0804 & 0.9130 & & 5.78960 & \\
\hline
\end{tabular}

Table 3: Fuzzy paired-wise comparisons matrix according to heat source $(\mathrm{C} 1)$

\begin{tabular}{|l|l|l|l|l|l|l|l|l|}
\hline & $\begin{array}{l}\text { Thermal } \\
\text { Lab }\end{array}$ & $\begin{array}{l}\text { Electrical } \\
\text { lab }\end{array}$ & $\begin{array}{l}\text { Cad } \\
\text { Lab }\end{array}$ & $\begin{array}{l}\text { Cam } \\
\text { Lab }\end{array}$ & $\begin{array}{l}\text { Electronics } \\
\text { Lab }\end{array}$ & Product & $\begin{array}{l}\text { 5th root } \\
\text { of } \\
\text { product }\end{array}$ & $\begin{array}{l}\text { Normalized } \\
\text { weights } \\
\text { (NW) }\end{array}$ \\
\hline $\begin{array}{l}\text { Thermal } \\
\text { Lab }\end{array}$ & 1 & $1 / 3$ & $1 / 7$ & $1 / 5$ & $1 / 7$ & 0.00136 & 0.26714 & 0.03792 \\
\hline $\begin{array}{l}\text { Electrical } \\
\text { lab }\end{array}$ & 3 & 1 & 3 & 5 & 7 & 315.00000 & 3.15982 & 0.44852 \\
\hline Cad Lab & 7 & $1 / 3$ & 1 & 3 & 5 & 35.00000 & 2.03617 & 0.28903 \\
\hline
\end{tabular}




\begin{tabular}{|l|l|l|l|l|l|l|l|l|}
\hline Cam Lab & 5 & $1 / 5$ & $1 / 3$ & 1 & 3 & 1.00000 & 1.00000 & 0.14195 \\
\hline $\begin{array}{l}\text { Electronics } \\
\text { Lab }\end{array}$ & 7 & $1 / 7$ & $1 / 5$ & $1 / 3$ & 1 & 0.06667 & 0.58181 & 0.08259 \\
\hline $\begin{array}{l}\text { Sum of } \\
\text { Column }\end{array}$ & 23.0000 & 2.0095 & 4.6762 & 9.5333 & 16.1429 & & 7.04494 & \\
\hline Sum x NW & 0.8722 & 0.9013 & 1.3515 & 1.3532 & 1.333168049 & & 5.81140 & \\
\hline
\end{tabular}

Table 4: Criteria C1 Results

\begin{tabular}{|l|l|}
\hline CI & 0.202849 \\
\hline RI & 1.21 \\
\hline CR & 0.167644 \\
\hline
\end{tabular}

Table 5: Fuzzy paired-wise comparisons matrix according to Design (C2)

\begin{tabular}{|l|l|l|l|l|l|l|l|l|}
\hline & $\begin{array}{l}\text { Thermal } \\
\text { Lab }\end{array}$ & $\begin{array}{l}\text { Electrical } \\
\text { lab }\end{array}$ & $\begin{array}{l}\text { Cad } \\
\text { Lab }\end{array}$ & $\begin{array}{l}\text { Cam } \\
\text { Lab }\end{array}$ & $\begin{array}{l}\text { Electronics } \\
\text { Lab }\end{array}$ & Product & $\begin{array}{l}\text { 5th root of } \\
\text { product }\end{array}$ & $\begin{array}{l}\text { Normalized } \\
\text { weights (NW) }\end{array}$ \\
\hline Thermal Lab & 1 & $1 / 9$ & $1 / 7$ & $1 / 5$ & $1 / 3$ & 0.00106 & 0.25405 & 0.03147 \\
\hline Electrical lab & 9 & 1 & 3 & 5 & 9 & 1215.00000 & 4.13919 & 0.51272 \\
\hline Cad Lab & 7 & $1 / 3$ & 1 & 3 & 7 & 49.00000 & 2.17791 & 0.26978 \\
\hline Cam Lab & 5 & $1 / 5$ & $1 / 3$ & 1 & 5 & 1.66667 & 1.10757 & 0.13719 \\
\hline $\begin{array}{l}\text { Electronics } \\
\text { Lab }\end{array}$ & 3 & $1 / 9$ & $1 / 7$ & $1 / 5$ & 1 & 0.00952 & 0.39424 & 0.04883 \\
\hline $\begin{array}{l}\text { Sum of } \\
\text { Column }\end{array}$ & 25.0000 & 1.7556 & 4.6190 & 9.4000 & 22.3333 & & 8.07295 & \\
\hline Sum $\mathbf{x W}$ & 0.7867 & 0.9001 & 1.2461 & 1.2896 & 1.090645057 & & 5.31323 & \\
\hline
\end{tabular}

Table 6: Criteria C2 Results

\begin{tabular}{|l|l|}
\hline $\max$ & 5.31323 \\
\hline CI & 0.078308 \\
\hline RI & 1.21 \\
\hline CR & 0.064717 \\
\hline
\end{tabular}

Table 7: Fuzzy paired-wise comparisons matrix according to ventilation (C3)

\begin{tabular}{|l|l|l|l|l|l|l|l|l|}
\hline & $\begin{array}{l}\text { Thermal } \\
\text { Lab }\end{array}$ & $\begin{array}{l}\text { Electrical } \\
\text { lab }\end{array}$ & $\begin{array}{l}\text { Cad } \\
\text { Lab }\end{array}$ & $\begin{array}{l}\text { Cam } \\
\text { Lab }\end{array}$ & $\begin{array}{l}\text { Electronics } \\
\text { Lab }\end{array}$ & Product & $\begin{array}{l}\text { 5th root of } \\
\text { product }\end{array}$ & $\begin{array}{l}\text { Normalized } \\
\text { weights (NW) }\end{array}$ \\
\hline $\begin{array}{l}\text { Thermal } \\
\text { Lab }\end{array}$ & 1 & $1 / 7$ & $1 / 5$ & $1 / 5$ & $1 / 3$ & 0.00190 & 0.28574 & 0.03682 \\
\hline $\begin{array}{l}\text { Electrical } \\
\text { lab }\end{array}$ & 7 & 1 & 3 & 5 & 9 & 945.00000 & 3.93628 & 0.50725 \\
\hline Cad Lab & 5 & $1 / 3$ & 1 & 3 & 7 & 35.00000 & 2.03617 & 0.26239 \\
\hline Cam Lab & 5 & $1 / 5$ & $1 / 3$ & 1 & 5 & 1.66667 & 1.10757 & 0.14273 \\
\hline $\begin{array}{l}\text { Electronics } \\
\text { Lab }\end{array}$ & 3 & $1 / 9$ & $1 / 7$ & $1 / 5$ & 1 & 0.00952 & 0.39424 & 0.05080 \\
\hline $\begin{array}{l}\text { Sum of } \\
\text { Column }\end{array}$ & 21.0000 & 1.7873 & 4.6762 & 9.4000 & 22.3333 & & 7.76000 & \\
\hline Sum x NW & 0.7733 & 0.9066 & 1.2270 & 1.3416 & 1.13462964 & & 5.38314 & \\
\hline
\end{tabular}

Table 8: Criteria C3 Results

\begin{tabular}{|l|l|}
\hline Lmax & 5.38314 \\
\hline CI & 0.095786 \\
\hline RI & 1.21 \\
\hline CR & 0.079162 \\
\hline
\end{tabular}


Table 9: Fuzzy paired-wise comparisons matrix according to Electrical equipment (C4)

\begin{tabular}{|l|l|l|l|l|l|l|l|l|}
\hline & $\begin{array}{l}\text { Thermal } \\
\text { Lab }\end{array}$ & $\begin{array}{l}\text { Electrical } \\
\text { lab }\end{array}$ & $\begin{array}{l}\text { Cad } \\
\text { Lab }\end{array}$ & $\begin{array}{l}\text { Cam } \\
\text { Lab }\end{array}$ & $\begin{array}{l}\text { Electronics } \\
\text { Lab }\end{array}$ & Product & $\begin{array}{l}\text { 5th root of } \\
\text { product }\end{array}$ & $\begin{array}{l}\text { Normalized } \\
\text { weights (NW) }\end{array}$ \\
\hline Thermal Lab & 1 & $1 / 5$ & $1 / 3$ & $1 / 7$ & $1 / 9$ & 0.00106 & 0.25405 & 0.04898 \\
\hline Electrical lab & 5 & 1 & 3 & $1 / 3$ & $1 / 5$ & 1.00000 & 1.00000 & 0.19279 \\
\hline Cad Lab & 3 & $1 / 3$ & 1 & $1 / 5$ & $1 / 7$ & 0.02857 & 0.49112 & 0.09468 \\
\hline Cam Lab & 7 & 3 & $1 / 3$ & 5 & $1 / 3$ & 11.66667 & 1.63452 & 0.31511 \\
\hline Electronics Lab & 9 & 5 & $1 / 7$ & 3 & 1 & 19.28571 & 1.80737 & 0.34844 \\
\hline Sum of Column & 25.0000 & 9.5333 & 4.8095 & 8.6762 & 1.7873 & & 5.18705 & \\
\hline Sum x NW & 1.2244 & 1.8379 & 0.4554 & 2.7340 & 0.622765227 & & 6.87447 & \\
\hline
\end{tabular}

Table 10: Criteria C4 Results

\begin{tabular}{|l|l|}
\hline Lmax & 6.87447 \\
\hline CI & 0.468618 \\
\hline RI & 1.21 \\
\hline CR & 0.387287 \\
\hline
\end{tabular}

Table 11: Fuzzy paired-wise comparisons matrix according to Utilization (C5)

\begin{tabular}{|l|l|l|l|l|l|l|l|l|}
\hline & $\begin{array}{l}\text { Thermal } \\
\text { Lab }\end{array}$ & $\begin{array}{l}\text { Electrical } \\
\text { lab }\end{array}$ & $\begin{array}{l}\text { Cad } \\
\text { Lab }\end{array}$ & $\begin{array}{l}\text { Cam } \\
\text { Lab }\end{array}$ & $\begin{array}{l}\text { Electronics } \\
\text { Lab }\end{array}$ & Product & $\begin{array}{l}\text { 5th root } \\
\text { of } \\
\text { product }\end{array}$ & $\begin{array}{l}\text { Normalized } \\
\text { weights (NW) }\end{array}$ \\
\hline Thermal Lab & 1 & 5 & 7 & 5 & 1 & 175.00000 & 2.80936 & 0.36515 \\
\hline Electrical lab & $1 / 5$ & 1 & 3 & 1 & $1 / 5$ & 0.12000 & 0.65439 & 0.08505 \\
\hline Cad Lab & $1 / 7$ & $1 / 3$ & 1 & $1 / 3$ & $1 / 7$ & 0.00227 & 0.29588 & 0.03846 \\
\hline Cam Lab & $1 / 5$ & 3 & 3 & 5 & $1 / 5$ & 1.80000 & 1.12475 & 0.14619 \\
\hline $\begin{array}{l}\text { Electronics } \\
\text { Lab }\end{array}$ & 1 & 5 & 7 & 5 & 1 & 175.00000 & 2.80936 & 0.36515 \\
\hline $\begin{array}{l}\text { Sum of } \\
\text { Column }\end{array}$ & 2.5429 & 14.3333 & 21.0000 & 16.3333 & 2.5429 & & 7.69374 & \\
\hline Sum x NW & 0.9285 & 1.2191 & 0.8076 & 2.3878 & 0.928522198 & & 6.27153 & \\
\hline
\end{tabular}

Table 12: Criteria C5 Results

\begin{tabular}{|l|l|}
\hline Lmax & 6.27153 \\
\hline CI & 0.317882 \\
\hline RI & 1.21 \\
\hline CR & 0.262712 \\
\hline
\end{tabular}

Table 13: Fuzzy paired-wise comparisons for total criteria together

\begin{tabular}{|l|l|l|l|l|l|l|}
\hline COMPANY & $\begin{array}{l}\text { Heat } \\
\text { source }\end{array}$ & Design & Ventilation & $\begin{array}{l}\text { Electrical } \\
\text { equip }\end{array}$ & Utilization & $\begin{array}{l}\text { Priority } \\
\text { Weights (Pwi) }\end{array}$ \\
\hline NWj & 0.59769 & 0.1493 & 0.15186 & 0.05314 & 0.04805 & \\
\hline Thermal Lab & 0.03791973 & 0.0314689 & 0.036821932 & 0.048977093 & 0.3651492 & 0.05310239 \\
\hline Electrical lab & 0.44852314 & 0.5127232 & 0.507253203 & 0.19278772 & 0.0850548 & 0.092320634 \\
\hline Cad Lab & 0.28902563 & 0.2697783 & 0.262392879 & 0.094681637 & 0.038457 & 0.422580307 \\
\hline Cam Lab & 0.14194586 & 0.1371948 & 0.142727673 & 0.315114746 & 0.1461898 & 0.150946478 \\
\hline Electronics Lab & 0.08258563 & 0.0488349 & 0.050804312 & 0.348438804 & 0.3651492 & 0.188853064 \\
\hline
\end{tabular}


Table 14: Final results of ranking indexes using FAHP

\begin{tabular}{|c|c|c|c|c|c|c|c|c|c|c|}
\hline \multirow[b]{2}{*}{$\begin{array}{l}\text { Sl } \\
\text { No }\end{array}$} & \multirow{2}{*}{$\begin{array}{l}\text { Evaluation } \\
\text { of Green } \\
\text { concept } \\
\text { "HOWs" }\end{array}$} & \multirow{2}{*}{$\begin{array}{l}\text { Crisp } \\
\text { Weights }\end{array}$} & \multicolumn{5}{|c|}{ Evaluation of Green concept } & \multirow[b]{2}{*}{ C.I. } & \multirow[b]{2}{*}{ R.I. } & \multirow{2}{*}{$\begin{array}{l}\text { Inconsi } \\
\text { stency } \\
(\%)\end{array}$} \\
\hline & & & $\begin{array}{l}\text { Thermal } \\
\text { Lab }\end{array}$ & $\begin{array}{l}\text { Electrical } \\
\text { lab }\end{array}$ & $\begin{array}{l}\mathrm{Cad} \\
\mathrm{Lab}\end{array}$ & $\begin{array}{l}\text { Cam } \\
\mathrm{Lab}\end{array}$ & $\begin{array}{l}\text { Electronics } \\
\text { Lab }\end{array}$ & & & \\
\hline 1 & Heat source & 0.59769 & 0.03792 & 0.44852 & 0.28903 & 0.14195 & 0.08259 & 0.202849 & 1.12 & $18.11 \%$ \\
\hline 2 & Design & 0.1493 & 0.03147 & 0.51272 & 0.26978 & 0.13719 & 0.04883 & 0.078308 & 1.12 & $6.99 \%$ \\
\hline 3 & Ventilation & & & & & & 0.05 & 786 & 1.12 & $55 \%$ \\
\hline 4 & $\begin{array}{l}\text { Electrical } \\
\text { equip }\end{array}$ & 0.05314 & 0.04898 & 0.19279 & 0.09468 & 0.31511 & 0.34844 & 0.468618 & 1.12 & $41.84 \%$ \\
\hline \multirow[t]{3}{*}{5} & Utilization & 0.04805 & 0.36515 & 0.08505 & 0.03846 & 0.14619 & 0.36515 & 0.317882 & 1.12 & $28.38 \%$ \\
\hline & Overall Score & & 0.05310 & 0.09232 & 0.42258 & 0.15095 & 0.18885 & & & \\
\hline & Rank & & 5 & 4 & 1 & 3 & 2 & & & \\
\hline
\end{tabular}

\section{CONCLUSIONS}

Nowadays, succeeding to increase of laboratory usage activities and the importance of heat emission level by various activities like using of equipment's, power panels, co2 emission etc, the educational institutions has become more facilitate the laboratory operations and reduces the time by purchasing modern equipment's. The purpose of this study is to identify and select best green conditional laboratory based on FAHP calculations. In this paper the five alternative laboratories in an educational institution were selected. For calculation and ranking the five criteria of heat source, ventilation, design, electrical equipment's and utilization were considered. Due to the uneven scale of judgments and failure to adequate handle the inherent vagueness and carelessness in pairwise comparison process by AHP is criticized. So Fuzzy AHP method is developed to overcome all the shortcomings and respect to ambiguity and complexity of human decision making process.

The research results can be summarized as following items: cad lab with (0.4226) and electronics lab with $(0.05310)$ have the most priority weights and thermal lab $(0.05310)$ has the lowest weight. The most important criteria based on experts judgments in selecting the green conditional laboratory are respectively the heat source $(0.597)$ and ventilation (0.15186), design (0.1493), electrical equipment (0.05314) and utilization (0.1493). Table 8 results shows that cad lab has the highest ranking in heat source, ventilation, and design criteriawith thermal lab has the lowest rating between all the criteria. Future research can be used more comprehensive criteria for evaluating the green conditional laboratory. Other laboratories which haven't referred in this article can be cited for more accurate ranking. For subsequent studies, using other multi criteria decision making methods can be proposed to rank the institutional laboratories more properly.

\section{REFERENCES}

[1]. Meysanshaverdi, mahasaakbari , sajademamipour , Using Fuzzy multi criteria decision making approach for ranking the web browsers, International journal of economics and management sciences vol. 1, No. 8, 2012, pp. 72-86.

[2]. Soonhusoh , A decision making model for evaluating third-party logistics providers using fuzzy analytic hierarchy process, African journal of business management vol. 4(3), pp. 339-349, march 2010.

[3]. SarfarazHashemkhaniZolfani ,NahidRezaeiniya , MortezaPourhossein , Edmundas KazimierasZavadskas, decision making on advertisement strategy selection based on life cycle of products by applying fahp and topsis grey, InzinerineEkonomika-Engineering Economics, 2012, 23(5), 471-484

[4]. Yun-Chin Chen, Jun-Yuan Kuo and Bang-Ting Luo , Applying Fuzzy Analytic Hierarchy Process and Grey Relation Analysis to Evaluate the Supply Chain Performance of the Wafer Testing House, American Journal of Applied Sciences 8 (12): 1398-1403, 2011.

[5]. Oliver Meixner, fuzzy AHP group decision analysis and its application for

the evaluation of energy sources, Institute of Marketing and Innovation.

[6]. A. R. Ismail , S. Singh and C. W. Goh , Thermal Comfort In A Closed Air Conditioned Ict Laboratory At Ungku Omar Polytechnic Malaysia, 2010pp. 555-563. 